\title{
LIBRI RICEVUTI E RECENSIONI
}

R. E. Marshak, Riazzudin e C. P. RYAN - Theory of Weak Interactions in Particle Physics. WileyInterscience, A division of John Wiley and Sons, Inc., New York, London, ete., 1969; p. XIV-761; s. $285 /-$.

Il libro di Marshak, Riazzudin e RYAN, si propone di dare un quadro il più completo possibile delle interazioni deboli nella fisica delle particelle elementari. Di un libro del genere si sentiva abbastanza la mancanza. Le interazioni deboli costituiscono infatti una branca assai vasta della fisica delle particelle e la teoria di tali interazioni, senza poter dirsi definitiva, ha già delle sue caratteristiche ben precise. Lo sviluppo di tale teoria negli ultimi anni è stato notevole. Ciò nonostante, esposizioni generali dell'argomento si trovano spesso confinate in "lectures notes" od in articoli di rassegna. Il libro di MARSHAK et al. copre quindi un vuoto notevole nella lettera- tura sulle particelle elementari e lo fa con eccezionale cura. Gli sviluppi tecnici sono discussi molto dettagliatamente ed il volume può considerarsi come un ottimo libro di consultazione sull'argomento.

Il modo di presentazione è tale da renderlo consigliabile anche a ricercatori non esperti delle interazioni deboli.

I Capitoli I e II sono dedicati ad un'introduzione storica e matematica sull'argomento, con particolare riguardo alle simmetrie delle interazioni forti $\left(S U_{3} \times S U_{3}\right.$ ecc. $)$. Il Capitolo III è dedicato ai decadimenti leptonici. Il Capitolo IV tratta dei decadimenti semileptonici con $\Delta S=0$ ed il Capitolo $V$ tratta quelli con $\Delta S=0$. La fisica del sistema $\mathrm{K}_{0}, \overrightarrow{\mathrm{K}}_{0}$ e la violazione di $C P$ sono discussi in maniera molto chiara e completa. Il Capitolo VI discute i decadimenti non leptonici ed infine il Capitolo VII è dedicato all'ipotesi dell'esistenza di un bosone $\mathrm{W}$, che medi le interazioni deboli.

F. STROCCHI 